\title{
On the Probable Cause of the Discrepancies between Hipparcos and VLBI Pleiades Distance Measurements
}

\author{
Ernst Karl Kunst \\ Im Spicher Garten 5, Königswinter, Germany \\ Email: ernstkunst@aol.com
}

Received 22 September 2014; revised 19 October 2014; accepted 12 November 2014

Copyright (C) 2014 by author and Scientific Research Publishing Inc.

This work is licensed under the Creative Commons Attribution International License (CC BY). http://creativecommons.org/licenses/by/4.0/

c) (i) Open Access

\begin{abstract}
It will show, a recent extension of special relativity on the grounds of a novel concept of velocity, which also predicts the speed of transversal motions on the plane of the sky to increase with enduring observation time, to fully explain the differences of the observational results of the former experiments referring to the distance of the Pleiades from Earth.
\end{abstract}

\section{Keywords}

Pleiades, Discrepancies in Distance Measurements, Duality of Velocity Dependent on One-Way or Two-Way Measurement, Transversal Motions on the Plane of the Sky, Duration of Observation

\section{Introduction}

It is widely known that the orbiting observatory Hipparcos (High-Precision Parallax Collecting Satellite), launched in 1989 by ESA, among others also measured the distance to the open cluster Pleiades with $118.3 \pm 3.5$ pc (later publications e.g.: $120.2 \pm 1.5$ pc), where pc denotes parsec (e.g. [1]). Thereby the satellite measured the apparent position of some well-known cluster stars from different points of Earth's orbit relative to "fixed" distant stars (trigonometric parallax). These measurements covered four years and revealed very small shifts of star positions against the celestial background, being much more precise than ground-based techniques. The latter methods had constantly and correspondingly shown the Pleiades' distance to Earth to be about $133.5 \pm 1.2 \mathrm{pc}$ and, thus, to be at odds with the Hipparcos results. Therefore, since the publication of the Hipparcos Catalogue in 1997 this discrepancy has been considered as an unresolved problem.

But very recently a new measurement by using radio interferometry has been published by a cooperation of ten radio telescopes ranging over the whole Earth such that this VLBI (Very Long Base-Line Interferometry) 
array acts equivalently to a telescope the size of Earth (e.g. [2]). After a year and a half of observations of four star systems and four stars of the cluster, the network determined a distance of $136.2 \pm 1.2 \mathrm{pc}$; whereby only the far-away quasar J0347+2339 has been used as radio beacon. It is obvious that this result neatly complies with the pre-Hipparcos distance $133.5 \pm 1.2 \mathrm{pc}$, mainly derived by non-trigonometric methods. Therefore, the Hipparcos distance now is widely considered to be in error although according to the authors "no obvious systematic errors seem to be present in the obtained results" [1], i.e. the inconsistency between the original Hipparcos and the VLBI distance measurements prevails and the debate regarding the distance to the Pleiades is still open, also since the Hipparcos mission supposedly represents the most complete and exact astrometric survey of the sky and of the Pleiades cluster.

On the other hand, VLBI referenced to an essentially stationary quasar and using the above array of widely separated radio telescopes this gives the resolution of a telescope the size of Earth—as already mentioned. Thus, the latter global telescope essentially simultaneously could observe the apparent motion of oppositely positioned stars of the cluster. Furthermore, one should notice that to derive the cluster's absolute parallax the uncertainties of each target star's position with respect to the center of the cluster have been included, i.e. the binary orbit distances have been converted into a single cluster distance or to rephrase this: the apparent motions of the target stars obviously have been referenced to the stationary center of the Pleiades cluster. This implies that all measured apparent motions at opposite positions of Earth's orbit are referenced to the center of the cluster. In contrast to this very special VLBI method, Hipparcos referenced the apparent motion of each target star to some "fixed" star individually such that the measured parallaxes are absolute, i.e. the result of a global solution over the whole sky.

\section{A Difference of Observation Time of Physical Interest}

The above mentioned novel concept of special relativity and the associated modification of the Lorentz transformation among others propose the existence of an absolute rest frame of nature $\Sigma_{\mathrm{oo}}$ in the form of the spacefabric of symmetric Minkowski-space, indicated through the CMB, implying a duality of the speed of light as well as of inertial velocity in dependence on one-way or two-way measurement, respectively [3]. Accordingly will a far-away motion orthogonal to the line of sight with velocity $v_{0}$ along length $d$ on the plane of the sky be observed as the vector product of the diverging coordinate differences of space and time, respectively, in the order of

$$
\Delta x_{01(\mathrm{v} \top) 1-\text { way }}=\left|v_{0} \gamma_{0}^{2} \sqrt{\Delta t_{d}}\right| \cdot\left|\frac{v_{0}}{c}\left(c \Delta t_{01}\right)\right| \cdot \sin \frac{\pi}{2}=v_{0}^{2} \gamma_{0}^{2} \sqrt{\Delta t_{d}},
$$

where by angular dimension $\pi / 2$ is referred to the angle enclosing the reverse of the vector of the light reaching the observer along the line of sight and $d$ and, furthermore, the angle of the vector of the motion along $d$ relative to the line of sight is running backwards from $\pi / 2$ to zero (derivation see [3]). Furthermore, $\Delta t_{01}=1$, whereas velocity $v_{0}$ and Lorentz factor $\gamma_{0}$ denote the respective value relative to the absolute rest frame of nature or the $\mathrm{CMB}$, i.e. in this case $v_{\mathrm{o}(\mathrm{CMB})}=370 \mathrm{~km} \cdot \mathrm{s}^{-1}$ of the Sun. In [3] also has been shown that Equation (1) satisfactorily explains the discrepancies between measured and expected values e.g. of the position of the pulsar B1952+32, the running rejuvenation or apparent accelerative behavior of the crab nebula and the measured considerably different time dilations of quasars and supernovae with about the same redshift, i.e. distance to Earth. It is clear that Equation (1) must be valid in the case of the Hipparcos and VLBI measurements too, since owing to the Sun's motion relative to the CMB, Earth's orbit projected on the plane of the sky will deliver different, i.e. increasing values, in dependence on time of observation. Hence, one convincingly can proceed from the assumption that the discrepancies between the Hipparcos and VLBI measurements also should be due to the relativistic phenomenon expressed through Equation (1).

But from the previous it is obvious that only one of the parallax measurements fulfills the requirements of the coordinate difference of observation time $\Delta t_{d}$ in the right-hand square root of Equation (1), that is motion from a point at rest relative to the observer alongside distance $d$ transversal to the latter. With the parallax measurements referenced to the Pleiades center the VLBI time of observation clearly fulfills the above demand. Note that other than VLBI centered at the Pleiades, Hipparcos' four years lasting parallax measurements cover the whole cluster and, thus, also the parallaxes at the opposite sides of Earth's orbit. This implies observation time of VLBI either to be doubled or of Hipparcosless by half, to make both measurements equivalent in the sense of 
$\sqrt{\Delta t_{d}}$ in Equation (1). With this clarification in advance, doubling VLBI observation time one can put the ratio.

$$
\frac{\text { Parallax }_{\text {Hipparcos }}}{\text { Parallax }_{\mathrm{VLBI}}}=\left(\frac{\Delta t_{\text {Hipparcos }}}{2 \cdot \Delta t_{\mathrm{VLBI}}}\right)^{\frac{1}{2}}=\frac{\text { distance }_{\mathrm{VLBI}}}{\text { distance }_{\text {Hipparcos }}}
$$

of the different distance estimations of both parallax measurements, where $\Delta t$ in the mid-ratio denotes the respective observation time. This leads in accord with Equation (1) to the further ratio

$$
\frac{\text { distance }_{\text {VLBI }}}{\text { distance }_{\text {Hipparcos }}}=\frac{370^{2}\left(1-\frac{370^{2}}{c^{2}}\right)^{-1} \sqrt{4 y}}{370^{2}\left(1-\frac{370^{2}}{c^{2}}\right)^{-1} \sqrt{2 \times 1.5 y}}=1.1547
$$

( $y$ in the right-hand ratio denotes year), whereas the respective ratio of the observationally derived VLBI-distance $136.2 \pm 1.2 \mathrm{pc}$ and Hipparcos-distance $118.3 \pm 3.5$ pc delivers:

$$
\frac{\text { distance }_{\mathrm{VLBI}}}{\text { distance }_{\text {Hipparcos }}}=\frac{136.2 \pm 1.2 \mathrm{pc}}{118.3 \pm 3.5 \mathrm{pc}}=1.1513 \pm 0.0232 \text {. }
$$

Hence, the latter experimental ratio $1.1513 \pm 0.0232$ of the above derived distances from measurements of the trigonometric parallax of Earth's orbit at the distance of the Pleiades cluster with differently long observation times seems to be in good accordance with the theoretically derived value 1.1547 of Equation (2a), implying the above discussed discrepancies in distance measurements to owe their existence the relativistic effect Equation (1).

\section{Concluding Remarks}

The above introduced extension of special relativity [3] seems to fully resolve the discrepancy between Hipparcos and VLBI parallax measurements, additionally to the previously mentioned applications of the former theory. Thus, this additionally shows that physical notions derived from longer lasting observations of motions on the plane of the sky are full of uncertainties and thus either in the reach of the Milky Way Galaxy classical methods or beyond the sphere of the latter redshift measurements alone or in association with luminosity observations must be called upon for sufficiently reliable distance estimations as well.

\section{References}

[1] Robichon, N., et al. (1999) Open Clusters with Hipparcos. Astronomy \& Astrophysics, 345, 471-484.

[2] Melos, C., et al. (2014) A VLBI Resolution of the Pleiades Distance Controversy. Science, 345, 1029-1032. http://dx.doi.org/10.1126/science.1256101

[3] Kunst, E.K. (2014) Is the Kinematics of Special Relativity Incomplete? Natural Science, 6, 226-247. http://dx.doi.org/10.4236/ns.2014.64027 
Scientific Research Publishing (SCIRP) is one of the largest Open Access journal publishers. It is currently publishing more than 200 open access, online, peer-reviewed journals covering a wide range of academic disciplines. SCIRP serves the worldwide academic communities and contributes to the progress and application of science with its publication.

Other selected journals from SCIRP are listed as below. Submit your manuscript to us via either submit@scirp.org or Online Submission Portal.
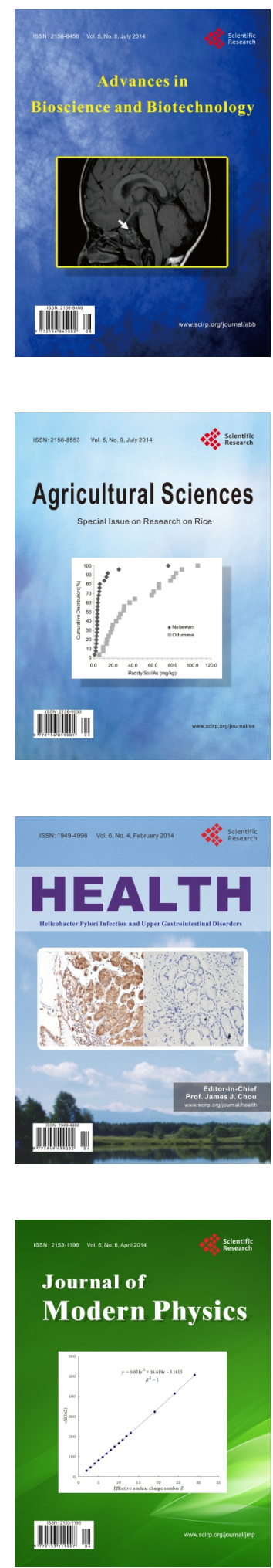
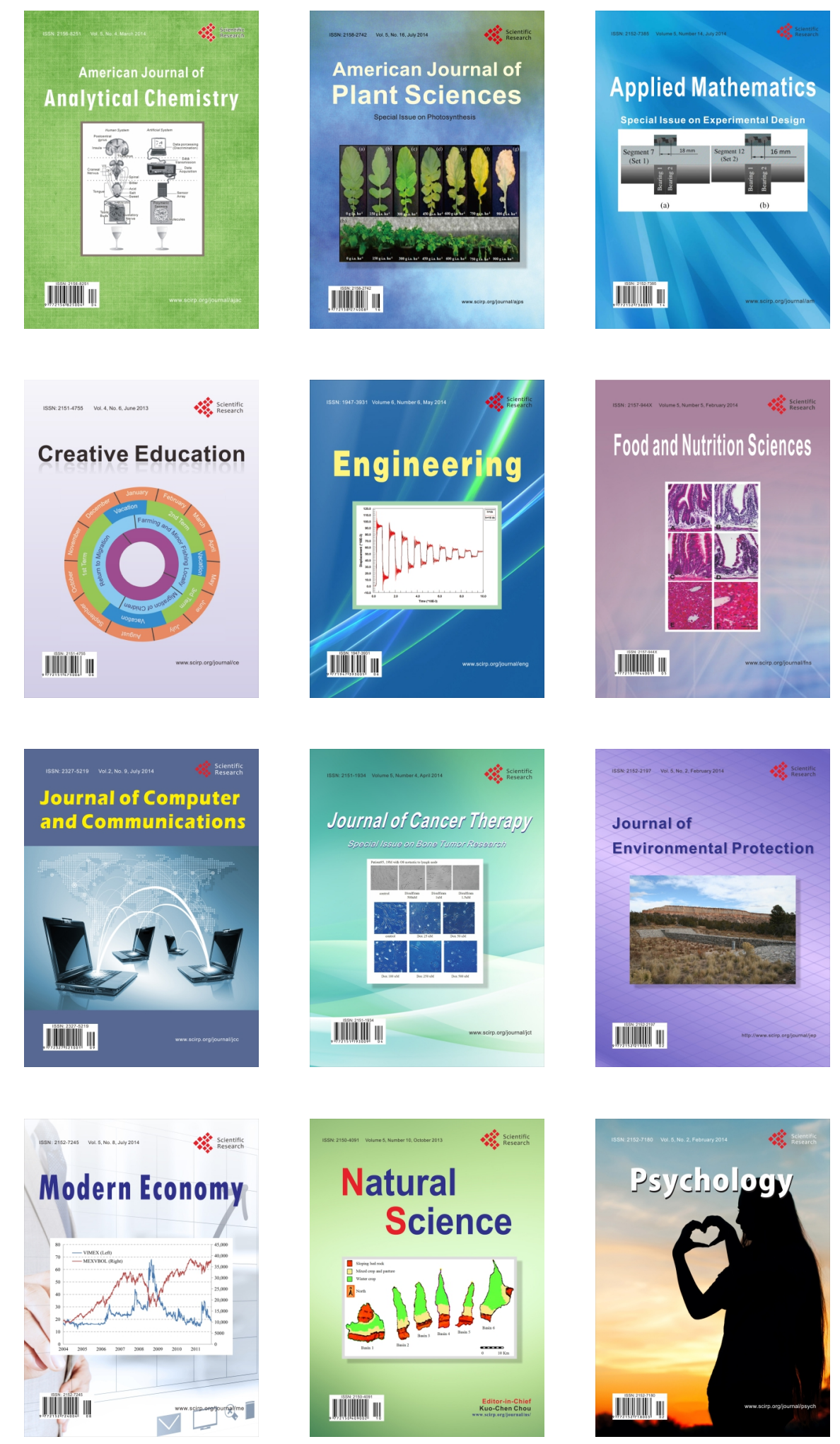